\title{
Historical Perspectives on Management of Acute Myocardial Infarction
}

\author{
Zhen Vin Lee and Bashir Hanif
}

\section{$1.1 \quad$ Introduction}

In 1977, the first successful coronary angioplasty was performed by Andreas Gruentzig using a double-lumen balloon catheter. This pivotal event spurred major advances in the field of percutaneous coronary intervention (PCI) over the subsequent four decades, including in the setting of myocardial infarction (MI) where primary PCI is now the established gold standard therapy. Nonetheless, although reperfusion is the cornerstone of management of acute MI, the role of various adjunctive therapies also needs to be recognized as these have had a substantial influence in improving both morbidity and mortality. This chapter serves to revisit the key historical milestones that have helped shape the modern management of acute MI.

\subsection{Angina Pectoris as a Clinical Entity}

In 1772, the eminent English physician, William Heberden, described what would later become known as angina pectoris in the most apt manner [1]:

'But there is a disorder of the breast marked with strong and peculiar symptoms, considerable for the kind of danger belonging to it, and not extremely rare, which deserves to be mentioned more at length. The seat of it, and sense of strangling, and anxiety with which it is attended, may make it not improperly be called angina pectoris. They who are afflicted with it, are seized while they are walking, (more especially if it be up hill, and soon after eating) with a painful and most disagreeable

Z.V. Lee $(\bowtie)$

University Malaya Medical Centre, Kuala Lumpur, Malaysia

e-mail: zhenvin@ummc.edu.my

B. Hanif

Tabba Heart Institute, Karachi, Pakistan 
sensation in the breast, which seems as if it would extinguish life, if it were to increase or continue; but the moment they stand still, all this uneasiness vanishes...'

As early as 1850, Richard Quain published a comprehensive review on fatty diseases of the heart, describing an observed association between coronary artery sclerosis and myocardial scars. Subsequently, the theory of 'coagulation necrosis' was coined by Cohnheim, who wrote in 1882, that 'the occlusion of a coronary artery, in case it does not prove fatal, leads to the destruction of the contractile substance of that portion of the heart, which is fed by the affected artery, and afterwards to the formation of so-called myocarditic indurations' [2].

Later, advances in imaging technology would make it possible to objectively identify narrowing (sclerosis) of the coronary arteries and actively demonstrate the presence of myocardial ischaemia allowing correlation between symptoms of the various ischaemic heart disease syndromes and the underlying anatomic and physiologic abnormalities [2]. Thus was born our understanding that at least in simplistic terms, restriction of blood flow in the epicardial coronary arteries led to symptomatic onset of disease.

\subsection{Development of the Electrocardiogram}

Over the late 1800 s to early 1900 s, cardiology witnessed a great technological breakthrough that was to have a major effect on the understanding of arrhythmia: the electrocardiograph (ECG). Physiologist Augustus Desiré Waller working in St. Mary's Hospital, London, recorded the first human surface ECG using the Lippmann capillary electrometer to deflect a light beam (Fig. 1.1). Waller had learnt that 'each beat of the heart gives an electric charge, beginning at one end of the organ and ending at the other'. He was convinced that he could measure these electromotive events from the skin surface and proceeded to do so with the electrometer connected between the left and right hands or between the front and back paws of his dog. The clinical significance of the electrocardiogram was not appreciated at the time. Waller himself said: 'I do not imagine that electrocardiography is likely to find any very extensive use in the hospital. It can at most be of rare and occasional use to afford a record of some rare anomaly of cardiac action' [3].

Another physiologist, Willem Einthoven, shares the honour with Waller of having a pivotal role in founding this new diagnostic modality. Einthoven recorded the first human ECG in 1892 using the Lippmann capillary electrometer. He initially indicated the four observed deflections with the characters A, B, C and D but later instead adopted the middle characters of the alphabet: P, Q, R, S and T. In 1902, he made the first direct recording of the true human ECG using a modified string galvanometer (Fig. 1.2) [4]. Although there was wide scepticism by the contemporary scientific community against his methods, Einthoven continued publishing, in 1913 described the Einthoven triangle as the basis for calculations of ECGs and introduced the bipolar electrode system. Classic rhythms were obtained and published. In 1924, he was awarded the Nobel Prize for Physiology and Medicine for his pioneering work in developing the string galvanometer [3]. 


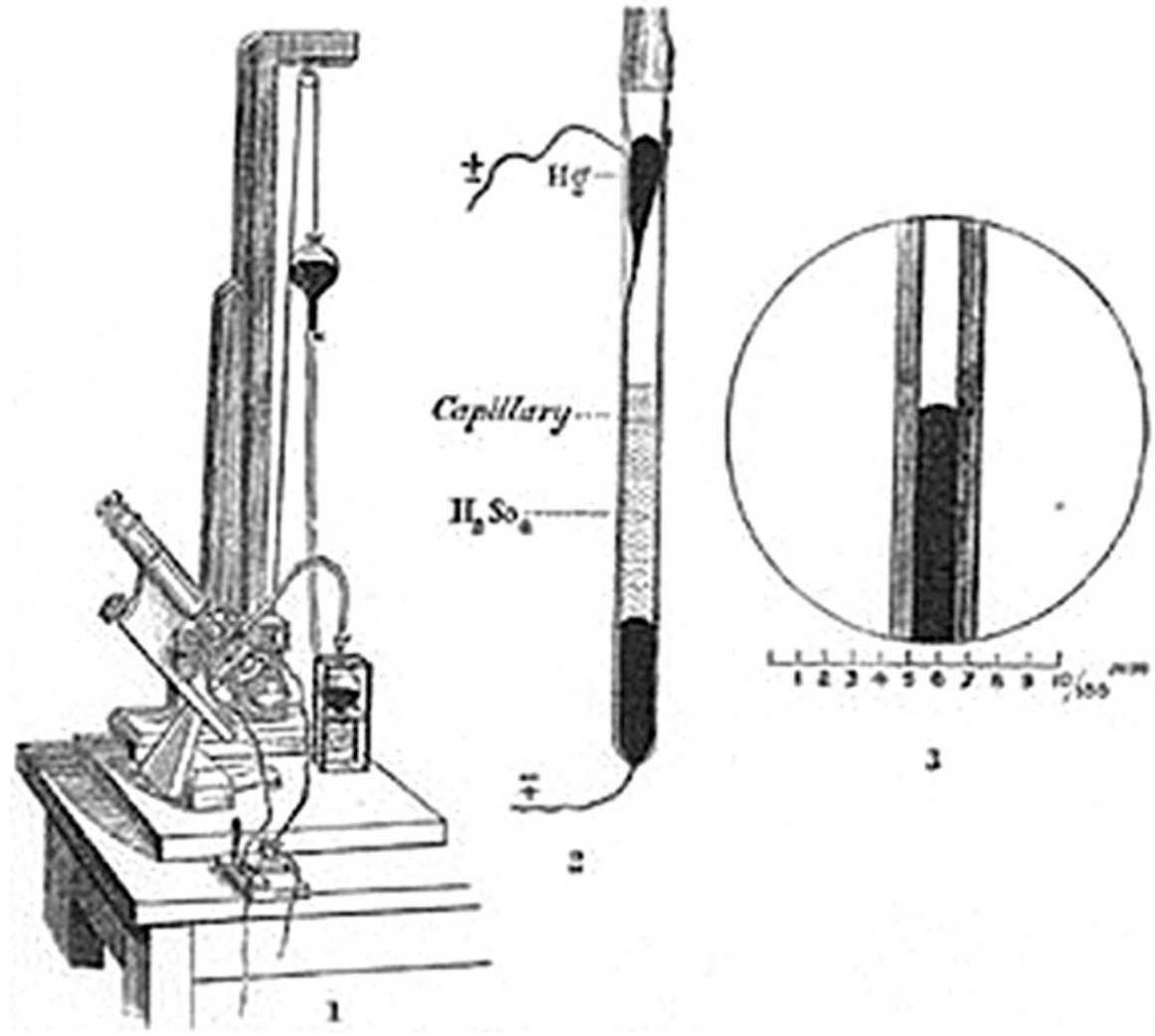

Fig. 1.1 Lippmann capillary electrometer (Source: Aquilina O. A brief history of cardiac pacing. Images Pardiatr Cardiol. 2006;8 (2): 17-81)

\subsection{Evolving Concepts in Pathophysiology of Myocardial Infarction}

Although, even in the nineteenth century, coronary thrombosis was recognized as a cause of death, it was predominantly regarded as a medical curiosity. On the basis of animal experiments involving ligation of a major coronary artery and of limited observations in human beings at necropsy, for many years the condition was considered to be immediately and universally fatal. However, in 1901, Krehl reported that coronary thrombosis did not always result in sudden death and that symptoms were more severe when arterial occlusion was sudden as opposed to progressive. He also recognized that MI may be complicated by ventricular aneurysm formation and myocardial rupture [4].

Once it became clear that survival from acute myocardial infarction (AMI) was possible, attention naturally began to be directed towards management. In his 1912 paper, Herrick stated that after AMI, 'the importance of absolute rest in bed for several days is clear'. This dictum would become the cornerstone of therapy for the 


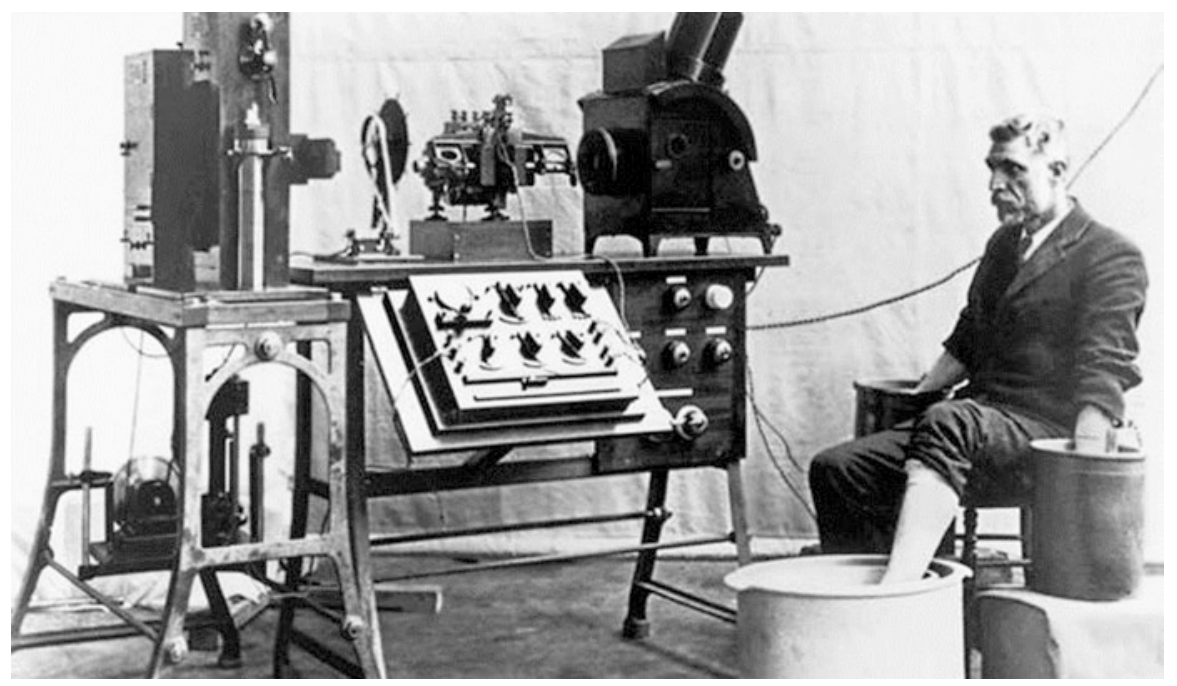

Fig. 1.2 ECG recording using a modified string galvanometer (Source: AlGhatrif M, Lindsay J. A brief review: history to understand fundamentals of electrocardiography. Journal of Community Hospital Internal Medicine Perspectives. 2012;2(1). Available from: doi:https://doi.org/10.3402/jchimp.v2i1.14383)

next half-century. Herrick also recognized that hope for restoration of the integrity of the damaged myocardium was possible where collateral blood supply developed. In the course of his work, Herrick also reported ECG findings consistent with acute MI, enabling the ECG to become a powerful diagnostic test in the recognition of this condition [4].

In the early 1920s, Wearn described the first significant series of patients with AMI, in all 19 of whom clinical-pathological correlations had been made. He recommended that 'every effort [be made] to spare the patient any bodily exertion' to prevent sudden death from cardiac rupture. In patients with pulmonary rales, fluid intake was restricted and digitalis given. Caffeine and camphor, two stimulants (vasopressors) then available, were used to prevent hypotension, syncope and heart block. Later that decade, Samuel Levine described a series of his own patients with acute MI. He identified the frequency of, and risk posed by, various cardiac arrhythmias. He recommended quinidine to treat ventricular tachycardia and intramuscular adrenaline to treat heart block and syncope [4].

By the 1930s and 1940s, as outcomes slowly started to improve, there was considerable debate about when in the course of the illness patients could be permitted to sit in a chair, use a commode, ambulate, be discharged from the hospital and ultimately resume their normal activities. In 1952, when Levine and Lown proposed the 'armchair treatment' of AMI, this suggestion, quite radical at the time, provoked heated debate. However, by the mid-twentieth century, it had become clear that although MI was now the most common cause of death in the industrialized world, concepts for post MI care needed to evolve. Cardiac rupture, although almost 
universally fatal, was relatively uncommon; absolute limitation of physical activity was not truly required; long-term bed rest might itself be associated with serious and occasionally fatal complications such as venous thromboembolism. As a consequence, practice gradually changed. Ambulation was accelerated and convalescence shortened; post-infarction rehabilitation made possible a more rapid return to regular lifestyle [4].

\subsection{Cardiopulmonary Resuscitation and External Defibrillation}

Coupled with advances in understanding of natural history of CAD and MI, Beck and colleagues in 1947 had resuscitated, by electric shock, a 14-year-old boy in whom ventricular fibrillation developed during surgery. Beck's group was also successful in correcting ventricular fibrillation, using open thoracotomy, in a 65-year-old physician with a myocardial infarction [5]. Soon after, Zoll introduced external defibrillation demonstrating that 'ventricular tachycardia and fibrillation' could be successfully terminated by externally applied electric countershock. This of course was not always successful, but it was recognized that early application of the shock was required to maximize efficacy and that cardiac monitoring provided opportunity for immediate recognition of cardiac arrest and identification of the arrhythmia [6]. A further advance observation was efficacy of mouth-to-mouth breathing, sternal compression and closed-chest electrical defibrillation in restoring normal cardiac function in some victims of ventricular fibrillation. It was this advance that triggered the interest in intensive care for myocardial infarction [7].

\subsection{The Coronary Care Unit}

The coronary care unit (CCU) is, perhaps, the single most important advancement in the treatment of MI and encompasses clinical application of four separate developments, namely, the appreciation of the importance of arrhythmias as the principal cause of early death in MI; ability to monitor the ECG continuously with the cathode-ray oscilloscope; development of closed-chest cardiac resuscitation; and delegation of the treatment of life-threatening arrhythmias, particularly ventricular fibrillation, to trained nurses in the absence of physicians [4].

Desmond Julian of the Royal Infirmary of Edinburgh is largely credited as the pioneer in the development of the CCU and wrote in his 1961 that 'Many cases of cardiac arrest associated with acute myocardial ischaemia could be treated successfully if all medical, nursing and auxiliary staff were trained in closed chest cardiac massage and if the cardiac rhythm of patients with MI were monitored by an ECG linked to an alarm system... Such units should be staffed by suitably experienced people throughout the 24 hours' [8]. Julian's colleagues in Edinburgh were 
unenthusiastic about his concept, and subsequently he moved to Australia where he launched a programme of continuous monitoring of patients who presented to Sydney Hospital with MI [9]. The first presentation of coronary care given to the British Cardiovascular Society was at the Autumn Meeting in 1964 when the Sydney experience was described [10].

It was clearly apparent that the new treatment technologies had to be used immediately to save lives. To achieve this goal, doctors had to abandon traditional notions of a nurse's limited role in clinical decision-making and transition to a system where the CCU nurse was able to implement therapeutic measures by herself without specific orders, including sometimes the definitive treatment for ventricular fibrillation. The CCU-inspired empowerment of nurses represented a critical first step in the evolution of team-based care that is such a conspicuous part of current-day cardiology practice [9].

With improving survival from MI, Myocardial Infarction Research Units (MIRU) were created in the United States, and a large programme of research was initiated into the investigation of the haemodynamic effects of myocardial infarction. It was quickly shown that the commonly used right atrial and central venous pressures provided an unreliable index of left-sided function. The introduction of the SwanGanz flow-guided catheter was a major advancement in the evaluation of cardiac performance in the CCU, allowed more precise delineation of the various haemodynamic subsets of patients with MI and facilitated their more rational treatment [10].

\subsection{Concept of Reduction of Infarct Size}

Left ventricular function emerged as a critical factor in the outcome in patients who were nursed in the CCU and in whom fatal primary arrhythmias were prevented. Infarct size became recognized as the major determinant of mortality and morbidity, and the concept was put forwards that even after the onset of infarction the quantity of damaged myocardium could be influenced by interventions designed to improve the balance between oxygen supply and demand in the jeopardized zone [4]. Preservation of left ventricular function became the major predictive factor of prognosis. The size of the infarct was then defined as the major determinant of mortality and morbidity. The use of injectable and oral beta blockers not only to treat arrhythmias but also to limit myocardial damage induced by the ischemic area arose as a therapeutic possibility. These drugs reduce oxygen consumption by the myocardium and enhance blood redistribution from the epicardium to the myocardium, diminishing the area of infarct and increasing survival [11].

\subsection{Advent of Thrombolysis and Role of Aspirin}

In the 1950s and 1960s, Fletcher and Verstraete pioneered the experimental use of streptokinase for thrombolysis. By the 1970s, Chazov and Rentrop were driving what would become a revolution in cardiology, demonstrating that intracoronary infusion of streptokinase could dissolve intracoronary thrombi, thereby limiting the 
infarct extension and size. This work was corroborated by the studies of De Wood et al. according to which $90 \%$ of patients with clinical findings of infarction and alterations in the ST segment had occlusive thrombi in the coronary arteries. The need for a direct intracoronary injection, however, was the major obstacle to its use. Intravenous infusion, which was easier and faster, quickly proved to be equivalent to the intracoronary infusion [11].

The ISIS-2 trial represented a significant milestone in rapid treatment of AMI with streptokinase and aspirin. This trial demonstrated that streptokinase and aspirin alone each produced a significant reduction in 5-week vascular mortality. The combination of streptokinase and aspirin was synergistic and markedly better than either treatment alone. The differences in vascular and in all-cause mortality produced by streptokinase and aspirin remained highly significant, even after a median of 15 months of follow-up [12]. This trial was followed by the GISSI study, which again proved the benefits of streptokinase and indicated that earlier treatment led to greater benefit [13].

Streptokinase though was known to have limitations, including propensity to induce hypotension, high rates of allergy, prolonged anticoagulant effect and development of neutralizing antibodies. It was clear that more efficacious and safer agents were needed. This paved the way for the GUSTO trial series which led to the introduction of differing thrombolytic regimens including the use of accelerated tissue plasminogen activator (t-PA) with intravenous heparin. Although bleeding remained a concern, accelerated t-PA given with intravenous heparin seemed to confer a survival benefit $[14,15]$. As agents were further refined, recombinant tissue-type plasminogen activator (rt-PA) was introduced and showed a significantly reduced 1-month mortality in a cohort of patients with suspected AMI who were randomized to receive either rt-PA plus heparin or placebo plus heparin. Whilst there was an excess of bleeding complications in the rt-PA group of patients, the incidence of stroke was similar [16]. The introduction of single-bolus injection of tenecteplase (a genetically engineered variant of alteplase with slower plasma clearance) would facilitate more rapid treatment of AMI both in hospital and in the community setting [17].

These results together with the low incidence of severe adverse effects made the use of thrombolytic agents unquestionable in the first hours of AMI. In addition to significantly reducing mortality, thrombolytic agents protect against associated morbid events, such as cardiogenic shock and heart failure, in a direct relation to the speed with which they are administered. In regard to adverse effects, thrombolytic therapy is related to a small and significant increase in the occurrence of cerebral strokes [11].

\subsection{Coronary Angiography and Percutaneous Revascularization}

Accurate depictions of the epicardial coronary arteries are present in the historical works of Leonardo da Vinci and Andreas Vesalius; however, smaller intramural branches were difficult to be demonstrated by simple dissection. Later, post-mortem injection techniques facilitated in-depth study of coronary circulation [18]. 
With the discovery of X-rays in 1895 by Wilhelm Röntgen, a new approach to the study of cardiac anatomy would, with time, become possible. These experiments were made possible by development of a technique for human right heart catheterization by the German physician Werner Forssmann who undertook catheterization of his own heart in 1929. Forssmann soon extended his experiments to include the intracardiac injection of contrast material. Forssmann's contributions together with the development of nontoxic contrast materials and steady advances in X-ray equipment and technique set the stage for the development of cardiac angiography and subsequently coronary arteriography [18].

In the 1950s, Mason Sones developed more selective coronary imaging. Whilst performing aortographic examinations in patients with rheumatic valvular disease, Sones discovered that some injections would preferentially fill one coronary artery and that this caused no apparent harm to the patient. After his studies of semi-selective coronary arteriography, Sones began to perform true selective coronary arteriography in 1958. Sones combined anatomic and physiologic considerations in developing his method of selective coronary arteriography and designed a preformed catheter with a tapered tip permitted selective entry into the coronary ostia but avoided complete obstruction of the coronary artery-an event much feared by earlier physicians who were not using tapered catheters. Furthermore, Sones introduced continual pressure monitoring combined with fluoroscopy to alert the operator to inadvertent complete obstruction of the coronary artery [18].

This has paved the way for the progressive advancement and modernization in the field of coronary angiography and angioplasty. Diagnostic catheters, guiding catheters, coronary guidewires, balloon catheters and coronary stents were invented and upgraded. Techniques of PCI were invented, modified and improved upon, details of which will be further elaborated in subsequent chapters of this handbook.

\subsection{Progress of Adjunctive Pharmacotherapy}

\subsubsection{Beta Blockade}

In 1984, the International Collaborative Study Group reported the benefits of timolol. Given as a bolus followed by oral maintenance, this agent was shown to reduce myocardial ischaemia and infarct size as measured by an accelerated reduction of ST-vector magnitude, a significant reduction of maximal cumulative creatine kinase release and significantly smaller changes in QRS-vector variables. Timolol was also associated with significant reductions in pain and was well tolerated overall [19]. Further studies demonstrated efficacy for other beta blockers, notably metoprolol and atenolol, which proved to be even more effective with markedly improved longer-term outcomes through to 1 year $[20,21]$. 


\subsubsection{ACE Inhibitors}

The role of angiotensin converting enzyme (ACE) inhibitors was established in a number of studies. The AIRE study found that ramipril resulted in a significant reduction in all-cause mortality as well as a significant reduction in the first validated outcome, namely, death, severe or resistant heart failure, MI or stroke in patients with clinical heart failure. These benefits were seen as early as 30 days [22] . Similarly, administration of captopril after AMI was shown to attenuate the process of progressive ventricular enlargement and was able to confer improvement in left ventricular (LV) systolic function in those patients where LV dysfunction was most marked [23, 24]. Later, the HOPE study would report that ramipril significantly reduced the rates of death, myocardial infarction and stroke in a broad range of highrisk patients who were not known to have a low ejection fraction or heart failure [25].

\subsubsection{HMG Co-A Reductase Inhibitors}

The 4S trial reported that in stable patients with angina or previous MI with elevated serum cholesterol, on a lipid-lowering diet, simvastatin significantly improved survival and reduced the risk of undergoing myocardial revascularization procedures and established the role of statins in management of coronary artery disease (CAD) [26]. Subsequent studies indicated similar advantage from other statins, even where serum cholesterol levels were in the normal or low range [27-29]. Later data would emerge indicating that early administration of statin may offer advantage in the early phase after onset of AMI.

\subsubsection{Thienopyridines}

Aggregating platelets have long been suspected of having an important role in the development of coronary thrombi, but it remained for the ISIS- 2 trial to show unequivocally the enormous effectiveness of aspirin which is a simple, welltolerated and inexpensive drug in reducing mortality in AMI [4].

The CAPRIE trial randomized 19,185 patients with atherosclerotic vascular disease manifested as either recent ischaemic stroke, recent MI or symptomatic peripheral arterial disease to either clopidogrel at dose of $75 \mathrm{mg}$ once daily or aspirin at dose of $325 \mathrm{mg}$ once daily. The authors reported that clopidogrel is more effective than aspirin in reducing the combined risk of ischaemic stroke, MI or vascular death and the overall safety profile of clopidogrel is at least as good as that of mediumdose aspirin [30].

In the PLATO trial, 18,624 patients who were admitted with an acute coronary syndrome (ACS), with or without ST-segment elevation, were randomized to receive either clopidogrel or ticagrelor, which is an oral, reversible, direct-acting 
inhibitor of the adenosine diphosphate receptor, P2Y12, that has a more rapid onset and more pronounced platelet inhibition than clopidogrel. Ticagrelor was found in the trial to have significantly reduced the rate of death from vascular causes, MI or stroke without an increase in the rate of overall major bleeding but with an increase in the rate of non-procedure-related bleeding compared to clopidogrel [31] .

In the TRITON-TIMI 38 trial, 13,608 patients with moderate- to high-risk ACS with scheduled PCI were randomized to receive a new thienopyridine and prasugrel or to receive clopidogrel. It was found that prasugrel therapy was associated with significantly reduced rates of ischemic events, including stent thrombosis, but with an increased risk of major bleeding, including fatal bleeding. Overall mortality did not differ significantly between treatment groups [32].

\subsubsection{Glycoprotein Ilb/IIIa Inhibitors}

The EPIC trial randomized 2099 patients who were scheduled to undergo coronary angioplasty or atherectomy in high-risk clinical situations such as severe unstable angina, evolving AMI or high-risk coronary morphologic characteristics to either a bolus and an infusion of placebo, a bolus of a chimeric monoclonal-antibody Fab fragment (c7E3 Fab) directed against the platelet glycoprotein IIb/IIIa receptor and an infusion of placebo or a bolus and an infusion of c7E3 Fab. The investigators concluded that ischemic complications of coronary angioplasty and atherectomy were reduced with the usage of c7E3 Fab, although the bleeding complications were increased [33].

The RAPPORT trial demonstrated that in a cohort of patients suffering from AMI of less than $12 \mathrm{~h}$ duration, who underwent primary percutaneous transluminal coronary angioplasty (PTCA), administration of abciximab resulted in a significant reduction in the incidence of death, reinfarction or urgent target vessel revascularization at 30 days compared to placebo albeit at a cost of higher bleeding rates. The 6-month primary end point however was the same for both treatment groups [34].

The EPILOG trial on the contrary was terminated early as abciximab, together with both low-dose and standard-dose heparin, significantly reduced the risk of acute ischemic complications compared to placebo and standard-dose heparin in 2792 patients who underwent either urgent or elective percutaneous coronary revascularization [35].

\section{Conclusion}

The last 120 years have witnessed considerable progress in our understanding and management of CAD and MI. Modern treatment algorithms have encompassed and refined techniques to achieve rapid reperfusion and salvage-threatened myocardium and enhance prospects for patient stability and recovery. 


\section{References}

1. Classics in cardiology: description of angina pectoris by William Heberden. Heart Views 2006;7:118-9.

2. Chopra HK, Nanda NC. Textbook of cardiology (a clinical \& historical perspective). New Delhi: Jaypee Brothers Medical Pub; 2013.

3. Aquilina O. A brief history of cardiac pacing. Images Pardiatr Cardiol. 2006;8(2):17-81.

4. Braunwald E. Evolution of the management of acute myocardial infarction: a 20th century saga. Lancet. 1998;352:1771-4. https://doi.org/10.1016/S0140-6736(98)03212-7.

5. Beck CF, Weckesser EC, Barry FM. Fatal heart attack and successful defibrillation: new concepts in coronary artery disease. JAMA. 1956;161:434-6. https://doi.org/10.1001/ jama.1956.62970050001008.

6. Zoll PM, Linenthal AJ, Zarsky LRN. Ventricular fibrillation-treatment and prevention by external electric currents. N Engl J Med. 1960;262:105-12. https://doi.org/10.1056/ NEJM196001212620301.

7. Julian DG. The evolution of the coronary care unit. Cardiovasc Res. 2001;51(4):621-4. https:// doi.org/10.1016/S0008-6363(01)00365-0.

8. Julian DG. Treatment of cardiac arrest in acute myocardial ischaemia and infarction. Lancet. 1961;2:840-4.

9. Fye WB. Resuscitating a circulation abstract to celebrate 50th anniversary of the coronary care unit concept. Circulation. 2011;124:1886-93. https://doi.org/10.1161/ CIRCULATIONAHA.111.033597.

10. Julian DG. The history of coronary care units. Br Heart J. 1987;57(6):497-502.

11. Sarmento-Leite R, Krepsky AM, Gottschall CAM. Acute myocardial infarction. One century of history. Arq Bras Cardiol. 2001;77:602-10. https://doi.org/10.1590/ S0066-782X2001001200011.

12. ISIS-2 Collaborative Group. Randomized trial of intravenous streptokinase, oral aspirin, both, or neither among 17187 cases of suspected acute myocardial infarction: ISIS-2. Lancet. 1988;2:349-60.

13. Gruppo Italiano per lo Studio Della Streptochinasi Nell' Infarto Miocardico (GISSI). Effectiveness of intravenous thrombolytic treatment in acute myocardial infarction. Lancet. 1986;327:397-402. https://doi.org/10.1016/S0140-6736(86)92368-8.

14. The GUSTO investigators. An international randomized trial comparing four thrombolytic strategies for acute myocardial infarction. The GUSTO trial. N Engl J Med. 1993;329:673-80. https://doi.org/10.1056/NEJM199309023291001.

15. The Global Use of Strategies to Open Occluded Coronary Arteries (GUSTO III) Investigators. A comparison of reteplase with alteplase for acute myocardial infarction. N Engl J Med. 1997;337:1118-23. https://doi.org/10.1056/NEJM199710163371603.

16. Anglo-Scandinavian Study of Early Thrombolysis (ASSET). Trial of tissue plasminogen activator for mortality reduction in acute myocardial infarction. Lancet. 1988;2:525-30.

17. Assessment of the Safety and Efficacy of a New Thrombolytic (ASSENT-2) Investigators. Single-bolus tenecteplase compared with front-loaded alteplase in acute myocardial infarction: the ASSENT-2 double-blind randomised trial. Lancet. 1999;354:716-22.

18. Fye WB. Coronary arteriography-it took a long time! Circulation. 1984;70:781-7. https://doi. org/10.1161/01.CIR.70.5.781.

19. The International Collaborative Study Group. Reduction of infarct size with the early use of timolol in acute myocardial infarction. N Engl J Med. 1984;310:9-15. https://doi.org/10.1056/ NEJM198401053100103.

20. The MIAMI Trial Research Group. Metoprolol in acute myocardial infarction (MIAMI). A randomized placebo controlled international trial. Eur Heart J. 1985;6:199-226. https://doi. org/10.1093/oxfordjournals.eurheartj.a061845. 
21. ISIS-1 (First International Study of Infarct Survival) Collaborative Group. Randomized trial of intravenous atenolol among 16027 cases of suspected acute myocardial infarction. Lancet. 1986;2:57-65.

22. The Acute Infarction Ramipril Efficacy (AIRE) Study Investigators. Effect of ramipril on mortality and morbidity of survivors of acute myocardial infarction with clinical evidence of heart failure. Lancet. 1993;342:821-8.

23. Pfeffer MA, Lamas GA, Vaughan DE, et al. Effect of captopril on progressive ventricular dilatation after anterior myocardial infarction. N Engl J Med. 1988;319:80-6. https://doi. org/10.1056/NEJM198807143190204.

24. Sharpe N, Smith H, Murphy J, et al. Early prevention of left ventricular dysfunction after myocardial infarction with angiotensin-converting enzyme inhibition. Lancet. 1991;337:872-6.

25. The Heart Outcomes Prevention Evaluation (HOPE) Study Investigators. Effects of an angiotensin-converting-enzyme inhibitor, ramipril, on cardiovascular events in high-risk patients. N Engl J Med. 2000;342:145-53. https://doi.org/10.1056/ NEJM200001203420301.

26. Pedersen TR, Kjekshus J, Berg K, et al. Randomized trial of cholesterol lowering in 4444 patients with coronary heart disease: the Scandinavian simvastatin survival study (4S). Lancet. 1994;344:1383-9.

27. Sacks FM, Pfeffer MA, Moye LA, et al. The effect of pravastatin on coronary events after myocardial infarction in patients with average cholesterol levels. The cholesterol and recurrent events trial (CARE). N Engl J Med. 1996;335:1001-9. https://doi.org/10.1056/ NEJM199610033351401.

28. The Long-Term Intervention with Pravastatin in Ischemic Disease (LIPID) Study Group. Prevention of cardiovascular events and death with pravastatin in patients with coronary heart disease and a broad range of initial cholesterol levels. N Engl J Med. 1998;339:1349-57. https://doi.org/10.1056/NEJM199811053391902.

29. Bybee KA, Lee JH, O'Keefe JH. Cumulative clinical trial data on atorvastatin for reducing cardiovascular events: the clinical impact of atorvastatin. Curr Med Res Opin. 2008;24:1217-29. https://doi.org/10.1185/030079908X292001.

30. CAPRIE Investigators. A randomized, blinded trial of clopidogrel versus aspirin in patients at risk of ischaemic events (CAPRIE). Lancet. 1996;348:1329-39.

31. Wallentin L, Becker RC, Budaj A, for the PLATO Investigators, et al. Ticagrelor versus clopidogrel in patients with acute coronary syndromes. N Engl J Med. 2009;361:1045-57. https:// doi.org/10.1056/NEJMoa0904327.

32. Wiviott SD, Braunwald E, McCabe CH, for the TRITON-TIMI 38 Investigators, et al. Prasugrel versus clopidogrel in patients with acute coronary syndromes. N Engl J Med. 2007;357:200115. https://doi.org/10.1056/NEJMoa0706482.

33. The EPIC Investigators. Use of a monoclonal antibody directed against the platelet glycoprotein IIb/IIIa receptor in high-risk coronary angioplasty. N Engl J Med. 1994;330:956-61. https://doi.org/10.1056/NEJM199404073301402.

34. Brener SJ, Barr LA. Burchenal JEB, et al. on behalf of the ReoPro and primary PTCA organization and randomized trial (RAPPORT) investigators. Randomized, placebo-controlled trial of platelet glycoprotein IIb/IIIa blockade with primary angioplasty for acute myocardial infarction. Circulation. 1998;98:734-41.

35. The EPILOG Investigators. Platelet glycoprotein IIb/IIIa receptor blockade and low-dose heparin during percutaneous coronary revascularization. N Engl J Med. 1997;336:1689-96. https://doi.org/10.1056/NEJM199706123362401. 
Open Access This chapter is licensed under the terms of the Creative Commons Attribution 4.0 International License (http://creativecommons.org/licenses/by/4.0/), which permits use, sharing, adaptation, distribution and reproduction in any medium or format, as long as you give appropriate credit to the original author(s) and the source, provide a link to the Creative Commons license and indicate if changes were made.

The images or other third party material in this chapter are included in the chapter's Creative Commons license, unless indicated otherwise in a credit line to the material. If material is not included in the chapter's Creative Commons license and your intended use is not permitted by statutory regulation or exceeds the permitted use, you will need to obtain permission directly from the copyright holder. 